\title{
Características de la expresión emocional en los escolares primarios y su manejo desde la perspectiva del personal docente-educativo
}

\author{
Characteristics of Emotional Expression in Primary School Children and Its \\ Management from the Perspective of the Teaching Staff
}

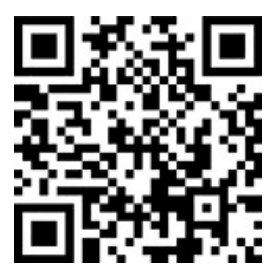

\author{
María Domingas Cassinda-Vissupe ${ }^{1}$ \\ Universidad Pedagógica de Bié \\ Bié, Angola \\ cassinda@uclv.cu \\ http://orcid.org/0000-0002-6187-8867 \\ Lisandra Angulo-Gallo² \\ Universidad Central Marta Abreu de Las Villas \\ Santa Clara, Cuba \\ lisandraa@uclv.cu \\ http://orcid.org/0000-0002-6533-041X \\ Vivian Margarita Guerra-Morales ${ }^{3}$ \\ Universidad Central Marta Abreu de Las Villas \\ Santa Clara, Cuba. \\ viviang@uclv.edu.cu \\ http://orcid.org/0000-0001-6323-7484
}

Recibido 10 de febrero de 2016 • Corregido 7 de noviembre de 2016 • Aceptado 2 de diciembre de 2016

\begin{abstract}
1 Profesora asistente de la Escuela Superior Pedagógica de Bié, Departamento de Ciencias de la Educación. Licenciada en Psicología, Máster en Psicología Médica. Actualmente realiza sus estudios doctorales en la Universidad Central "Marta Abreu" de Las Villas, Cuba. Ha publicado artículos y ha participado en eventos nacionales e internacionales relacionados con la psicoeducación, psicooncología y salud mental infanto-juvenil.

2 Profesora asistente de la Universidad Central "Marta Abreu" de Las Villas, Facultad de Ciencias Sociales, Departamento de Psicología. Licenciada en Psicología, Máster en Psicología Médica. Ha publicado artículos y ha participado en eventos nacionales e internacionales relacionados con la psicoeducación, psicooncología y salud mental infanto-juvenil, obtuvo, además, un premio de la Academia de Ciencias de Cuba (CITMA).

${ }^{3}$ Profesora titular de la Universidad Central "Marta Abreu" de Las Villas, Facultad de Ciencias Sociales, Departamento de Psicología. Doctora en Ciencias Psicológicas, Máster en Psicología Médica y Psicología de la Salud. Ha publicado artículos y ha participado en eventos nacionales e internacionales relacionados con la psicoeducación, psicooncología y salud mental infanto-juvenil, obtuvo, además, un premio de la Academia de Ciencias de Cuba (CITMA).
\end{abstract}


doi: http://dx.doi.org/10.15359/ree.21-1.19

URL: http://www.una.ac.cr/educare

CORREO: educare@una.cr

Resumen: La investigación tuvo como objetivo caracterizar la expresión emocional de los grupos escolares de la enseñanza primaria, los eventos vinculados a su manifestación y su manejo desde la perspectiva del personal docente-educativo. Se efectuó un estudio exploratorio descriptivo, con un muestreo no probabilístico, conformándose una muestra de 53 docentes pertenecientes a diversas escuelas de enseñanza primaria. Para la recogida de los datos se hizo uso del análisis de documentos, la entrevista y la observación; se empleó el análisis de contenido para su interpretación. El profesorado identificó un predominio de emociones placenteras en estudiantes, fundamentalmente la alegría. Las principales emociones displacenteras fueron la ansiedad y la ira, con poca alusión a emociones complejas como el orgullo, la culpa, la vergüenza o los celos. En el ámbito escolar las emociones displacenteras se asocian a las actividades y exigencias académicas, en relación con la familia se distinguieron los conflictos y poco apoyo recibido desde este contexto. Como factores generadores de emociones placenteras en la escuela se encuentran la aceptación grupal, profesionalismo docente y recreación, este último también presente en el ambiente familiar, en conjunto con el apoyo, dedicación y convivencia armónica. El personal docente declara no poseer la preparación teórica y metodológica necesaria para potenciar la educación emocional de sus estudiantes, aunque reconocen su importancia. Se destaca la necesidad de ofrecerle una formación más sólida que le permita conocer, comprender y regular sus propias emociones, así como potenciar el desarrollo afectivo de sus alumnos y alumnas, con la creación de un clima emocional que facilite el proceso de enseñanza aprendizaje.

Palabras claves: Emociones; expresión emocional; escolares; educación emocional.

\begin{abstract}
The research aimed to characterize the emotional expression of primary school children, events linked to its manifestation and its management from the perspective of the teaching staff. For this descriptive exploratory study, a non-probabilistic sample of 53 teachers from various primary schools was conformed. The study was carried out by using the analysis of documents, interviews and observation, and implementing the content analysis for interpretation. Teachers identified a predominance of pleasant emotions in school, mainly joy. The main unpleasant emotions were anxiety and anger. In the school context, the unpleasant emotions were associated with the activities and the academic demands; in the family, with conflict and little family support, fundamentally. The principal drivers of pleasant emotions in school are the group acceptance, teacher professionalism and recreation; the latter is also present in the family, together with the support, dedication and harmonious coexistence. The professor says not to possess the necessary theoretical and methodological training to direct the emotional education of their students, although they recognize its importance. It is very important to provide a more solid training that allows them to know, understand and regulate their emotions as well as enhance the emotional development of their students, creating an emotional environment which facilitates the process of learning.
\end{abstract}

Keywords: Emotions; emotional expression; emotional education.

\title{
Introducción
}

El estudio de las emociones resulta de vital trascendencia dentro del campo de la psicología, pues estas jugaron un papel decisivo en la supervivencia y la adaptación desde los orígenes de la vida del ser humano. Actualmente se reconoce su innegable potencial de riesgo/beneficio para la salud y el bienestar (Miguel-Toval, Cano-Vindel, 2002; Oblitas, 2010; Roca, 2014). 
doi: http://dx.doi.org/10.15359/ree.21-1.19

URL: http://www.una.ac.cr/educare

CORREO: educare@una.cr

Las emociones positivas promueven una organización cognitiva más abierta, flexible y compleja, lo cual posibilita que se ofrezcan, de forma creativa, mejores soluciones a los problemas. También se relacionan con la satisfacción de un enorme número de expectativas personales y sociales, ayudan a sobreponerse más rápido de sucesos negativos y desarmar los efectos perjudiciales que la emoción negativa puede acarrear. Además, se constata la existencia de una relación bidireccional entre las relaciones sociales y el afecto positivo, el cual induce a una conducta prosocial y, a su vez, la relación con los otros es una de las principales fuentes de afectos positivos (Férnández-Abascal, 2011).

En diversos estudios (Greco, 2010; Oros, 2011) efectuados con niñez de edad escolar se ha demostrado que, en esta etapa, las emociones positivas favorecen una apreciación más saludable de las dificultades y su afrontamiento de manera más funcional, incrementan aspectos cognitivos involucrados en la prevención de fracaso académico y fomentan actitudes más tolerantes y persistentes ante las frustraciones. Aunque los afectos positivos sean transitorios, contribuyen a la formación de procesos dinámicos permanentes con repercusión para la maduración personal (Fredrickson, 2013).

Por otra parte, son diversas las problemáticas de elevado nivel de impacto en la actualidad asociadas a una intensa emocionalidad negativa, experimentada por los sujetos de manera frecuente, como el abuso de sustancias, los robos, homicidios e intentos suicidas (García, 2013).

Los estados de ansiedad, depresión e ira pueden desencadenar diversos trastornos psicopatológicos, además de influir en el estado de salud general del sujeto, asociado a la aparición y pronóstico de muchas enfermedades (Piqueras, Ramos, Martínez y Oblitas, 2009; Vázquez y Hervás, 2009).

A partir de ello es posible apreciar la importancia que reviste la educación desde los diferentes contextos educativos y familiares en aras de promover habilidades emocionales para el desarrollo salutogénico desde la edad infantil.

Sin embargo, la escuela tradicionalmente ha privilegiado la enseñanza de contenidos puramente académicos, de ahí la necesidad de fortalecer la educación emocional en las aulas. Estudios como lo de Bisquerra (2012), Fernández-Berrocal, Berrios-Martos, Extremera, Augusto, 2012) han abordado esta temática planteando que ambos tipos de aprendizajes están inseparablemente interconectados y que se trata de una falsa dicotomía: no es necesario apostar por uno de ellos en detrimento del otro.

Específicamente, en la enseñanza escolar primaria se produce un cambio drástico en el trato verbal y la interrelación emocional que la persona adulta, especialmente el profesorado, establece con el alumnado, respecto a etapas anteriores (Abarca, 2003). Resulta de vital importancia que el profesorado reconozca y comprenda las emociones experimentadas por los escolares y las escolares y, desde su práctica, pueda influir positivamente en el desarrollo socio- 
doi: http://dx.doi.org/10.15359/ree.21-1.19

URL: http://www.una.ac.cr/educare

CORREO: educare@una.cr

afectivo de estos grupos. A partir del análisis anterior, se plantea el objetivo de caracterizar la expresión emocional de estudiantes de enseñanza primaria, los eventos vinculados a su manifestación y su manejo desde la perspectiva del personal docente-educativo.

\section{Referentes conceptuales}

Las emociones son procesos afectivos que emergen en la interacción del sujeto con el medio, y que conllevan a una expresión desde lo fisiológico, motor conductual expresivo y subjetivo experiencial, en la base de la valoración que realiza el sujeto del estímulo y el contenido vivencial que posea, agrado o desagrado, en integración con sus necesidades y motivaciones individuales (Guedes y Álvaro, 2010). Estos cambios presentan implicación para el funcionamiento biológico, psicológico y social del sujeto (Fernández Abascal, 2011; Pérez, 2014; Roca, 2014).

El reconocimiento de las manifestaciones emocionales de esctudiantes por parte del personal docente educativo constituye el eje central del estudio. Para ello se coincide con Sánchez-Aragón y Díaz-Loving (2009) al considerar la expresión emocional como una fuente de señales que permiten identificar y comprender las emociones experimentadas por el otro sujeto (gestos, expresiones faciales, comportamientos, posturas, verbalizaciones), además de considerarse sus posibilidades de expresividad o inhibición y ajuste a las normas o patrones culturales de expresión emocional.

El profesorado debe desarrollar su capacidad empática con el alumnado, reconociendo sus estados emocionales para tener así una relación cordial con este. Además de incidir en su desarrollo cognitivo, se requiere que contribuya a su desarrollo emocional, más aún en la etapa de la educación infantil y primaria, en la cual este se apropia de aquellos aprendizajes básicos para su futuro desempeño. Para ello, resulta clave la educación emocional, la cual es comprendida como un proceso educativo continuo y permanente que persigue optimizar el desarrollo integral de la persona, es, por tanto, una educación para la vida, además de ser una forma de prevención primaria (Bisquerra, 2012). Al respecto, diversas investigaciones han profundizado en la influencia del componente emocional en el éxito académico del alumnado y su adaptación escolar, aun cuando esta misma repercuta en prácticamente todos los ámbitos del proceso enseñanza aprendizaje (Enríquez, 2011; Fregoso, López, Navarro y Valadez, 2013; Jiménez y López, 2013).

\section{Aspectos metodológicos del estudio}

Para el desarrollo de la investigación se asumió un paradigma de investigación mixto, predominantemente cuantitativo. En cuanto a su alcance, fue empleado un tipo de estudio exploratorio-descriptivo. 
doi: http://dx.doi.org/10.15359/ree.21-1.19

URL: http://www.una.ac.cr/educare

CORREO: educare@una.cr

\section{Participantes}

El estudio se desarrolló en tres centros educativos rurales de enseñanza primaria de la provincia de Villa Clara. Para la selección del profesorado se efectuó un muestreo no probabilístico, considerando los criterios de selección siguientes:

\section{Criterios de inclusión}

- Que ejerzan como profesionales con vínculos con los centros de educación primaria en los cuales se desarrolla el estudio.

- Que tengan una experiencia, de cinco años como mínimo, de ejercicio de su profesión.

\section{Criterios de exclusión para los profesionales}

- Que no expresen su autorización mediante el consentimiento informado para participar en el estudio.

La muestra fue conformada por un total de 52 docentes: 49 del sexo femenino y 4 del sexo masculino. Las edades estuvieron comprendidas entre los 30 y 60 años. El 65,5\% posee más de 20 años en el ejercicio de su profesión, su formación académica es la siguiente: Licenciatura en Educación Primaria (34), Maestría en Ciencias de la Educación (10), Técnico o técnica en Auxiliar de Pedagogía (5), Técnicos o técnicas en Bibliotecología (2), Instructor o instructora de arte (1) y Maestría en la actividad física de la comunidad (1).

\section{Instrumentos}

\section{Entrevista psicológica estructurada}

La entrevista fue realizada con el objetivo de indagar acerca del conocimiento que posee el profesorado sobre la expresión emocional de sus estudiantes y las alternativas que emplean para su educación. Comprende aspectos como las manifestaciones (verbales, motoras, fisiológicas, subjetivas) de las emociones, factores asociados a su aparición, cómo trabaja la educación emocional en su práctica educativa, preparación recibida mediante su formación acerca del tema.

La entrevista, una vez elaborada y valorada por 3 especialistas en evaluación y diagnóstico psicológico, de la Carrera de Psicología de la UCLV, además de ser corregida y formalizada, se administró de forma piloto a 10 sujetos profesionales vinculados a la enseñanza primaria, para comprobar que las preguntas fueran fácilmente comprendidas y si motivaban las respuestas o aportes necesarios. La información obtenida en las entrevistas fue registrada mediante la grabación magnetofónica y posteriormente transcrita. 
doi: http://dx.doi.org/10.15359/ree.21-1.19

URL: http://www.una.ac.cr/educare

CORREO: educare@una.cr

\section{Observación}

La información recogida en las entrevistas fue triangulada mediante la observación. Con las observaciones en el aula se pretendía recoger las influencias directas e indirectas de la práctica educativa en la dimensión emocional. Para lograr este objetivo se solicitaron al profesorado varias sesiones de filmaciones en los diversos tipos de actividades que se desarrollan en la escuela: clases de Español, Matemática y Ciencias, receso, Educación Física, Computación y otras actividades informales, en las cuales se efectuó una observación por cada profesor o profesora. Se valoró la filmación como el medio más adecuado para realizar las observaciones. Por un lado, este medio contrarresta los efectos distorsionados producto de la implicación emocional de quien observa con las personas observadas (Hernández, Fernández y Baptista, 2010). Por otro lado, las filmaciones permiten categorizar los contenidos entre varios sujetos investigadores, y evitan, así, una interpretación errónea de la información por falta de comprensión del lenguaje, e incrementan la fiabilidad del análisis. Sin embargo, las filmaciones comportan el inconveniente de que pueden introducir sesgos en el comportamiento del profesorado y el alumnado, dada la deseabilidad de mostrar una imagen positiva, lo cual se consideró para el análisis de la información.

\section{Análisis de documentos oficiales}

La investigación documental es una técnica muy utilizada. Un documento en sentido amplio es cualquier objeto físico que constituya el registro de algún conocimiento, y puede ser escrito, audiovisual o arquitectónico, además de catalogarse como material interno o externo. Los documentos internos son todos aquellos que circulan dentro de determinadas organizaciones. Los externos, por su parte, son aquellos producidos por la organización para comunicarse con el exterior (Fernández-Ballesteros, 2004). En la investigación se revisaron planes de clases y programas de las asignaturas, así como los planes anuales de cada escuela. El objetivo perseguido fue explorar cómo se contemplan la dimensión emocional en los objetivos, contenidos, conceptos, procesos y actividades del centro y si estos contenidos se distribuyen de forma homogénea en las asignaturas, con un margen de coherencia en la conducción de dichos aspectos y su aplicación en la práctica del educador.

\section{Procedimiento}

La investigación se llevó a cabo en el período comprendido entre enero y febrero del 2015, con previa autorización de dichas instituciones para su despliegue, así como con el consentimiento informado al profesorado. Los encuentros se realizaron de manera individual, en horarios matutinos, en espacios donde existieran adecuadas condiciones para la realización de las entrevistas, respetando los principios éticos relacionados con la confidencialidad de la información.

6

María Domingas Cassinda-Vissupe, Lisandra Angulo-Gallo y Vivian Margarita Guerra-Morales 
doi: http://dx.doi.org/10.15359/ree.21-1.19

URL: http://www.una.ac.cr/educare

El procesamiento de los datos se realizó a través del método de análisis de contenido, que considera la posibilidad de analizar el significado de los datos cualitativos emergidos sin aplicar categorías exteriores o previas a la observación. Según Cabrera (2009), mediante este proceso, centrado en la manera en la que los individuos crean y atribuyen significaciones, se asumen procesos de interpretación, a partir de una lectura minuciosa de lo que dicen o escriben los actores para reconstruir los diferentes universos vivenciales. Con posterioridad, fueron agrupados en categorías, para proceder a su análisis cuantitativo, mediante el paquete estadístico SPSS, versión 21.0 para Windows. Se hizo uso de la estadística descriptiva, analizando la información mediante frecuencias absolutas y porcentuales, con el apoyo del diseño de tablas y gráficos que permitan una mejor visibilidad del contenido, así como de la estadística inferencial, específicamente la prueba Chi-Cuadrado de Pearson y el test exacto de Fisher.

\section{Resultados}

\section{Expresión emocional de escolares de la enseñanza primaria y eventos vinculados a su manifestación}

La totalidad de docentes de la muestra coincide en que las emociones placenteras predominan en los grupos escolares. El 81,1\% (43) menciona la alegría como la emoción placentera más frecuente, expresada mediante las manifestaciones que se muestran en la Figura 1:

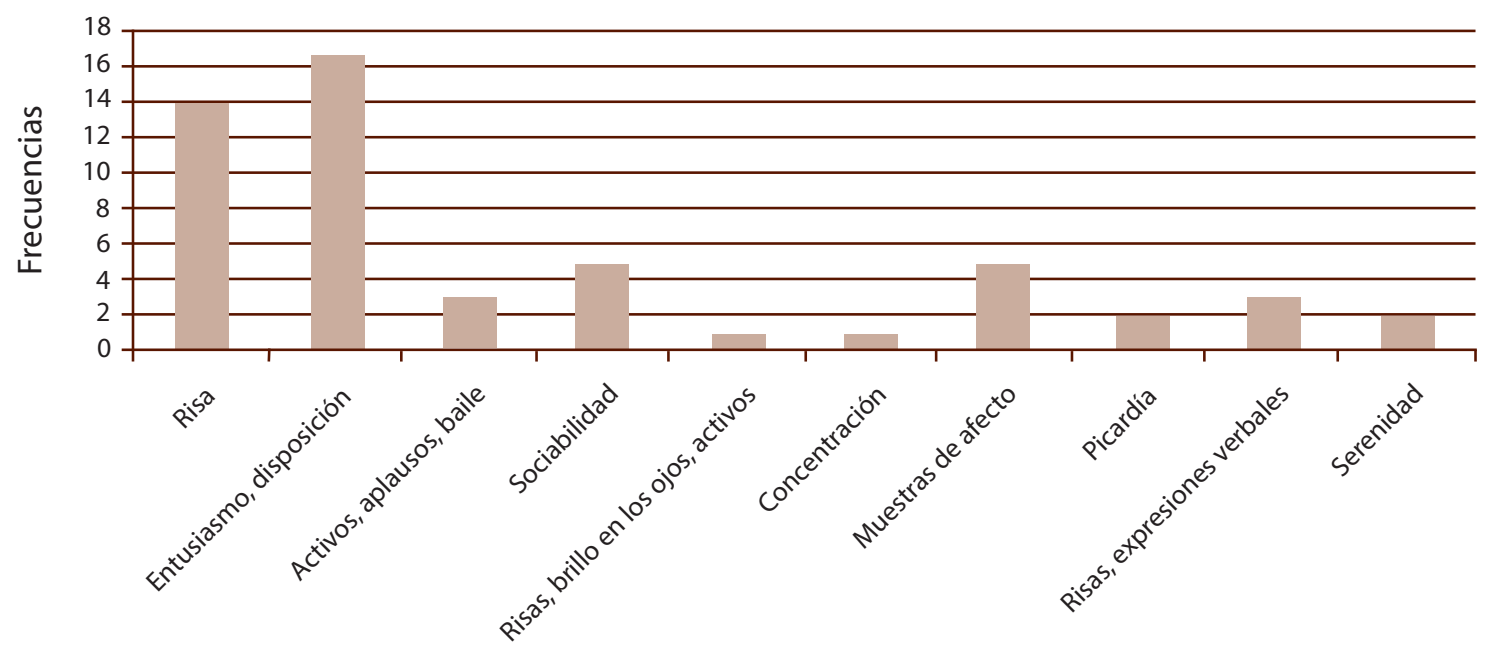

Manifestaciones

Figura 1: Expresiones de las emociones placenteras. 
doi: http://dx.doi.org/10.15359/ree.21-1.19

URL: http://www.una.ac.cr/educare

CORREO: educare@una.cr

Las emociones displacenteras que predominan en escolares, según el criterio del profesorado fueron: ira $(30,2 \%)$, tristeza $(30,2 \%)$ y ansiedad $(11,3 \%)$. Sus principales formas de expresión son las siguientes (Figura 2):

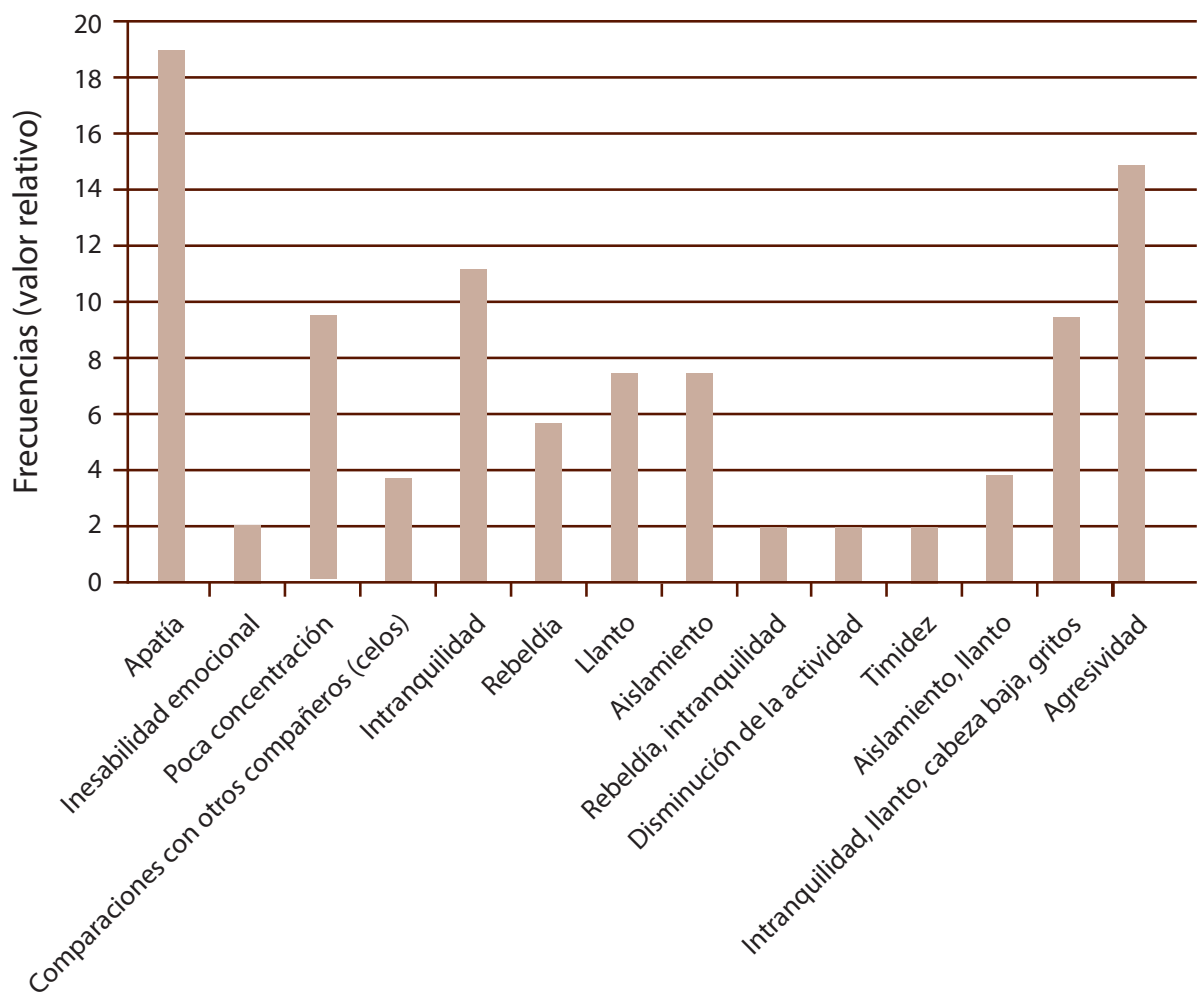

Manifestaciones

Figura 2: Expresiones de las emociones displacenteras.

Mediante la observación se pudo distinguir que las emociones vivenciadas, así como sus formas de expresión, variaban según el tipo de actividad desarrollado, predominando en muchas ocasiones estados displacenteros en actividades académicas y evaluativas. En las interacciones entre iguales se pudieron corroborar aspectos referidos por el personal docente relativos a una elevada incidencia de las manifestaciones de ira.

A partir de la distinción y diferenciación de las emociones, posteriormente se indaga en los eventos que, según el profesorado, le dan origen en los principales contextos socializadores: familia y escuela. 
doi: http://dx.doi.org/10.15359/ree.21-1.19

URL: http://www.una.ac.cr/educare

CORREO: educare@una.cr

En la familia emergieron como categorías principales, a partir de las verbalizaciones emitidas por el profesorado, el apoyo y dedicación de los padres y madres, así como la recreación (Tabla 1).

Tabla 1: Principales generadores de emociones placenteras en la familia

\begin{tabular}{|c|c|c|c|}
\hline$\%$ & Frecuencia & Categoría & Verbalizaciones \\
\hline 24.5 & 13 & $\begin{array}{l}\text { Apoyo y dedicación } \\
\text { de los padres }\end{array}$ & $\begin{array}{l}\text { "Ayuda de los padres, apoyo en las tareas, interés por sus resultados, } \\
\text { tiempo con la familia, estar con sus padres, preocupación por sus } \\
\text { intereses, cuando los ayudan, le forran las libretas, motivación de los } \\
\text { padres hacia el menor, preocupación y atención de los padres". }\end{array}$ \\
\hline 20.8 & 11 & Recreación & $\begin{array}{l}\text { "Que les saquen a pasear, salir a parques, fiestas, actividades familiares, } \\
\text { estar libres de la escuela, jugar". }\end{array}$ \\
\hline 9.4 & 5 & Estabilidad familiar & $\begin{array}{l}\text { "Estabilidad de la familia, padres casados, que los padres } \\
\text { permanezcan unidos, que los padres no cambien frecuentemente de } \\
\text { pareja". }\end{array}$ \\
\hline 7.5 & 4 & $\begin{array}{l}\text { Convivencia } \\
\text { familiar armónica }\end{array}$ & $\begin{array}{l}\text { "Buenas relaciones familiares, compartir actividades en familia, } \\
\text { adecuadas relaciones interpersonales entre los padres, no presenciar } \\
\text { peleas en el hogar". }\end{array}$ \\
\hline
\end{tabular}

Factores similares, en sentido inverso, fueron asociados a la presencia de emociones displacenteras, sobresaliendo la presencia de conflictos y violencia familiar (Tabla 2).

Tabla 2: Principales generadores de emociones displacenteras en la familia

\begin{tabular}{|c|c|c|c|}
\hline$\%$ & Frecuencia & Categorías & Verbalizaciones \\
\hline 22.6 & 12 & $\begin{array}{l}\text { Violencia y conflictos } \\
\text { familiares }\end{array}$ & $\begin{array}{l}\text { "Desacuerdos en la familia, problemas, discusiones de los padres, } \\
\text { peleas, maltrato, agresiones, que no se lleven bien". }\end{array}$ \\
\hline 17.0 & 9 & Poco apoyo familiar & $\begin{array}{l}\text { "Poco apoyo, inasistencia a reuniones, que no se ocupen de ellos, } \\
\text { no recibir ayuda en la realización de tareas, no atender a sus } \\
\text { necesidades, desinterés de los padres". }\end{array}$ \\
\hline 9.4 & 5 & Divorcio & "Separación, divorcio mal manejado, lejanía de los padres" \\
\hline 9.4 & 5 & $\begin{array}{l}\text { Estilos educativos } \\
\text { inadecuados }\end{array}$ & $\begin{array}{l}\text { "Que no les dejen jugar, no les complazcan, desconfianza sobre } \\
\text { las aptitudes del menor, poca tolerancia por parte de los padres, } \\
\text { muchas prohibiciones". }\end{array}$ \\
\hline
\end{tabular}


doi: http://dx.doi.org/10.15359/ree.21-1.19

URL: http://www.una.ac.cr/educare

CORREO: educare@una.cr

Se evidenció que dichas problemáticas, aunque tienen lugar en la familia, se reflejan en el desempeño del estudiantado en el aula.

En el contexto escolar, como se visualiza en las Tablas 3 y 4 , los factores estuvieron relacionados con la aceptación grupal, aspectos vinculados al profesionalismo docente y su relación con el alumnado y el tipo de actividades desarrolladas.

Tabla 3: Principales generadores de emociones placenteras en la escuela

\begin{tabular}{cccl}
\hline$\%$ & Frecuencia & Categorías & \multicolumn{1}{c}{ Verbalizaciones } \\
\hline 28,3 & 15 & Aceptación grupal & $\begin{array}{l}\text { "Atención, comprensión, buena relación del menor con sus } \\
\text { compañeros, aceptación de los otros". }\end{array}$ \\
28,3 & 15 & $\begin{array}{l}\text { Profesionalismo del } \\
\text { personal docente }\end{array}$ & $\begin{array}{l}\text { "Buenas clases, perfección en las actividades, que los profes } \\
\text { dominen el contenido, que los motiven, que se preocupen por sus } \\
\text { problemas, cuando se emplean recursos didácticos". }\end{array}$ \\
9,4 & 4 & $\begin{array}{l}\text { Actividades } \\
\text { recreativas }\end{array}$ & $\begin{array}{l}\text { "Danzas, educación física, huerto, receso, canciones en inglés, } \\
\text { saltar correr, estímulo, música para bailar, computación y bailes, } \\
\text { exposiciones círculos de interés, acampadas, salas de video, } \\
\text { televisión, chistes, dibujo, actividades extra-docentes". }\end{array}$ \\
\hline
\end{tabular}

Tabla 4: Principales generadores de emociones displacenteras en la escuela

\begin{tabular}{|c|c|c|c|}
\hline$\%$ & Frecuencia & Categorías & Verbalizaciones \\
\hline 37.7 & 20 & $\begin{array}{l}\text { Exigencias } \\
\text { académicas }\end{array}$ & $\begin{array}{l}\text { "Actividades y tareas que requieren esfuerzo, maestro exigente, } \\
\text { con autoridad, leer libros que no les gusta, exigencias del estudio, } \\
\text { escritura, clases extensas, mucho tiempo en el aula, clases que } \\
\text { no les gusta o contenidos que no dominan, llamarle la atención, } \\
\text { pruebas, hacer resúmenes, matemática". }\end{array}$ \\
\hline 13.2 & 7 & $\begin{array}{l}\text { Bajo rendimiento } \\
\text { académico }\end{array}$ & $\begin{array}{l}\text { "Poco dominio del contenido, dificultades en el contenido, } \\
\text { contenidos que no entienden, no saber una respuesta, obtener } \\
\text { malas notas". }\end{array}$ \\
\hline 11.3 & 6 & Violencia psicológica & "Burla por parte de otros, malas contestaciones de los maestros". \\
\hline 17,0 & 9 & Regaño, castigo & $\begin{array}{l}\text { "No les dejen hablar con su compañero, llamarle la atención, final } \\
\text { del juego, no dar la educación física, no poder correr, no tener clases } \\
\text { de computación, no realizar educación física o computación”. }\end{array}$ \\
\hline 9,4 & 5 & $\begin{array}{l}\text { Conflictos entre } \\
\text { iguales }\end{array}$ & $\begin{array}{l}\text { "Discusiones entre ellos mismos, desacuerdo entre estudiantes, que } \\
\text { otro se crea mejor, que no les presten los materiales sus amigos". }\end{array}$ \\
\hline
\end{tabular}


doi: http://dx.doi.org/10.15359/ree.21-1.19

URL: http://www.una.ac.cr/educare

CORREO: educare@una.cr

\section{Alternativas utilizadas por el personal docente para la educación de las emociones en sus escolares}

Durante las entrevistas realizadas se pudo constatar que la generalidad de los educadores y las educadoras posee desconocimiento de contenidos referentes a la educación de las emociones de escolares. El término educación emocional lo relacionan con educación de los valores: "se les enseña el compañerismo, a ser solidarios, la unidad, la amistad, la honestidad" o con la potenciación de emociones placenteras: "una forma de ayuda que oriente su estado emocional hacia lo positivo". Aunque en algunas respuestas se destacaron aspectos relevantes, más cercanos a los referidos en la bibliografía científica, como la forma de educar a la niñez, a partir del ejemplo (7,5\%), saber mostrar sus emociones y saber controlarlas (7,5\%).

En el Figura 3 se muestra la opinión que poseen acerca de la formación que recibieron para ejercer su profesión, respecto a la dimensión emocional.

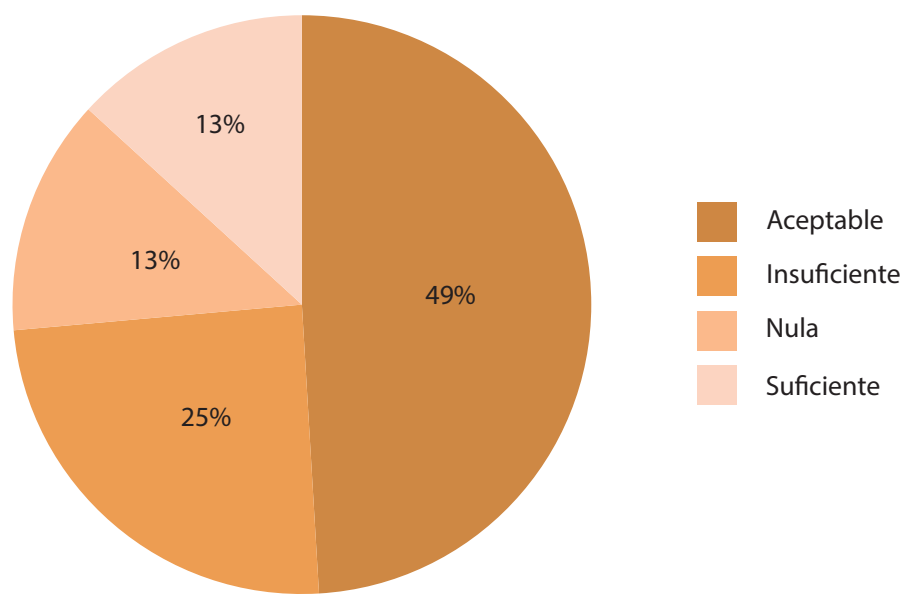

Figura 3: Formación profesional recibida por el profesorado.

En la formación post-graduada también se detectan insuficiencias, pues a pesar de la existencia de capacitaciones encaminadas a preparar al personal docente en el orden metodológico, político e ideológico, no resulta así en el orden afectivo. Solo un 15,1\% de educadores ha realizado cursos que le hayan aportado información para educar las emociones, $87 \%$ no han recibido preparación ninguna que haya favorecido el ejercicio profesional en estos aspectos. No se constataron diferencias significativas entre las distintas escuelas donde se desarrolló la investigación $(p=0.629)$ ni de acuerdo con la formación profesional $(p=0.523)$ o años de experiencia $(p=0.932)$ en relación con los criterios emitidos, lo cual demuestra la generalidad de esta problemática. 
doi: http://dx.doi.org/10.15359/ree.21-1.19

URL: http://www.una.ac.cr/educare

CORREO: educare@una.cr

Sin embargo, para el $98,1 \%$ de educadores y educadoras es muy importante el trabajo de la dimensión emocional en las escuelas, solo un 1,9\% considera que es una labor que solo ha de llevar a cabo la familia. En este sentido, la mayoría se pronunciaba hacia la necesidad de que exista correspondencia entre las influencias recibidas de la educación escolar y las provenientes del hogar.

Además, 36 sujetos destacan que el trabajo con el componente emocional queda reflejado en las directrices de los diseños curriculares de la educación primaria, solo el 30,2\% no comparte este criterio. En la revisión realizada de los programas de las asignaturas se constató que se incluyen contenidos de educación emocional, pero desde una perspectiva imprecisa y general, puesto que sus objetivos están centrados fundamentalmente en la comprensión de habilidades cognitivas necesarias para la superación de grados anteriores a los cursados, pero en la práctica profesional trabajan contenidos tales como: la laboriosidad, el compañerismo, las relaciones interpersonales y la comunicación, la aceptación de sí mismos y mismas, y de los otras personas como parte de la formación de valores.

Al abordarse la manera en que el profesorado trabaja la dimensión emocional en el contexto educativo, señalan que los aspectos tratados en su práctica profesionalson los que se muestran en la Figura 4.

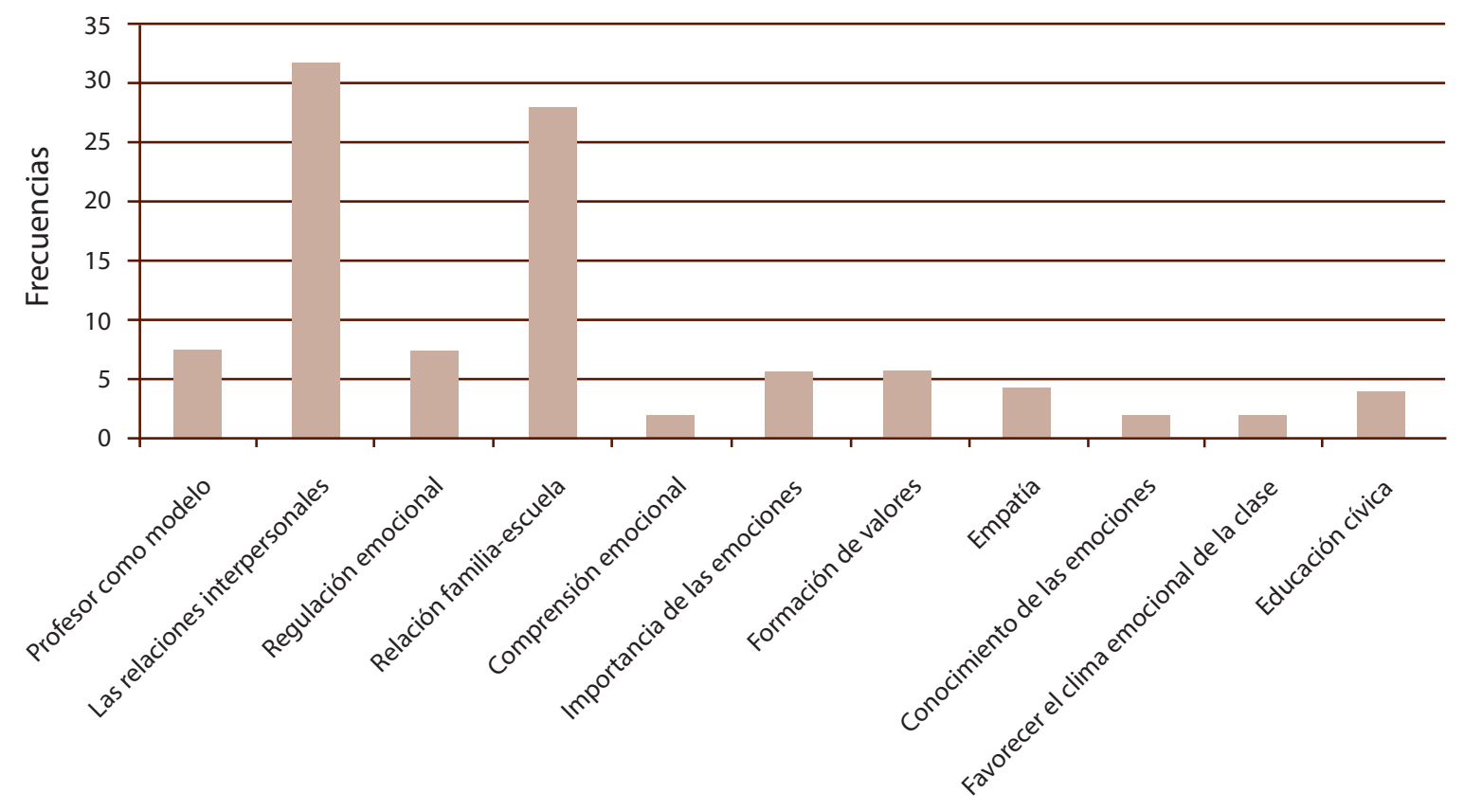

Manifestaciones

Figura 4: Aspectos relacionados con las emociones propuestas para trabajar por el profesorado en las aulas. 
Gran parte expone la carencia de conocimientos acerca de recursos prácticos y metodológicos para realizar un trabajo efectivo de las emociones, el 64,2\% manifiesta el desconocimiento de recursos metodológicos para trabajar la educación emocional. Ello demuestra que el sujeto profesional aborda estos contenidos de forma no intencional, puesto que no posee un dominio teórico-metodológico del tema. En este sentido, hacen alusión a la intuición y a la experiencia, puesto que a pesar de no contar con conocimientos propiamente desarrollados del tema, buscan soluciones ante las diferentes manifestaciones emocionales displacenteras de su estudiantado. Nadie hace alusión a alternativas que permitan, desde la práctica educativa, lograr en sus estudiantes una mejor comprensión de la emociones vivenciadas, no solo de cada quien, sino también en relación con las otras personas. A pesar de ello, mediante la observación, se evidenció el trabajo en estos componentes a partir del empleo de recursos literarios, fundamentalmente en la asignatura español (Cómo se sintió el personaje, por qué se sintió así, qué debe hacer para sentirse mejor, cómo te sentirías tú en su posición).

\section{Discusión}

El profesorado declaró un predominio de emociones placenteras en los grupos de escolares, fundamentalmente la alegría; y la ansiedad y la ira como las principales emociones displacenteras. Resulta interesante que fueron omitidas o consideradas con menor frecuencia otras emociones de carácter más complejo como la esperanza, el orgullo, la culpa y la vergüenza, etc. Es reconocido que las formas de expresión de estas son más inespecíficas (Etxebarria, 2009), sin embargo, resultan igualmente importantes, por lo que es necesario fomentar la preparación del profesorado para la comprensión y manejo de estas en el contexto escolar.

En este sentido, un hallazgo importante estuvo relacionado con el incremento de la agresividad en el estudiantado, como manifestación de la emoción displacentera ira, resultados coincidentes con otras investigaciones como las de Abarca (2003).

En relación con los eventos generadores de emociones placenteras y displacenteras en los principales contextos de socialización escolar, se pudo constatar una tendencia en el profesorado a asociar la recreación al afecto positivo y las exigencias académicas como causa de malestar emocional. Ello apunta hacia la necesidad de fomentar la motivación y el interés hacia las actividades docentes, para posibilitar un vínculo más estrecho entre los procesos cognitivos y afectivos.

Los conflictos y la falta de apoyo familiar también fueron reconocidos como principales factores de displacer, lo cual denota la repercusión que tiene este contexto para la salud mental infantil, y su reflejo en el espacio del aula. En este sentido, ha sido ampliamente demostrado que la interacción padres y madres-hijos e hijas puede facilitar el desarrollo afectivo del último grupo, o bien dificultar su florecimiento y expresión de las emociones (Henao y García, 2009; Richaud, 2010). 
doi: http://dx.doi.org/10.15359/ree.21-1.19

URL: http://www.una.ac.cr/educare

CORREO: educare@una.cr

Respecto al manejo de las emociones en el contexto educativo, se apreció que el personal docente reconoce la necesidad de trabajar la educación de las emociones en los niños y niñas de etapa escolar, aunque no posee la preparación teórica y metodológica suficiente para ello. Se destacan como aspectos fundamentales en su práctica educativa la promoción de relaciones interpersonales afectivas en el grupo, el vínculo familia-escuela, la figura del profesorado como modelo y la regulación de los estados emocionales displacenteros. Sin embargo, no se mencionan aspectos vinculados a la comprensión emocional y la empatía, aunque se observó un abordaje empírico y poco intencionado mediante su práctica educativa, donde el manejo de estas habilidades resulta un elemento esencial a fomentar en la escuela; similares deficiencias en la formación profesoral son detectadas por otros estudios (Fernández-Berrocal y Extremera, 2002).

\section{Conclusiones}

El estudio permitió concluir que los maestros y maestras estudiados son capaces de identificar las emociones placenteras y displacenteras en el alumnado, así como las situaciones asociadas a estas mismas, con mayores dificultades en la discriminación de aquellas de carácter más complejo. Además, reconocen la necesidad de potenciar la educación emocional en el contexto escolar, aunque se apreció una escasa preparación teórica metodológica para asumir esta labor.

A partir de estos resultados, se destaca la necesidad de ofrecer al profesorado una formación más sólida que le permita conocer, comprender y enseñar a regular sus propias emociones y las de sus estudiantes, para crear un clima emocional que facilite el proceso de enseñanza aprendizaje. Además, se reconoce la necesidad de continuar profundizando en el estudio realizado, al contemplar también la perspectiva del sujeto menor y de la familia en el abordaje de la expresión emocional infantil, donde se destaque la influencia que tiene este importante agente socializador en el desarrollo afectivo.

\section{Referencias}

Abarca, M. M. (2003). La educación emocional en la educación primaria: Currículo y práctica (Tesis doctoral). Universidad de Barcelona, España. Recuperado de http://hdl.handle.net/2445/42457

Bisquerra, R. (2012). Dela inteligencia emocional a la educación emocional. En R. Bisquerra (Coord.), ¿Cómo educar las emociones? La inteligencia emocional en la infancia y la adolescencia (pp. 24-34). Esplugues de Llobregat, Barcelona: Hospital Sant Joan de Déu. Recuperado de http://faros.hsjdben.org/adjuntos/2232.1-Faros\%206\%20Cast.pdf

Cabrera, I. (2009). El análisis de contenido en la investigación educativa: Propuesta de fases y procedimientos para la etapa de evaluación de la información. Revista Pedagogía Universitaria, 14(3), 71-93. Recuperado de http://cvi.mes.edu.cu/peduniv/index.php/ peduniv/article/view/501/495 
Enríquez, H. A. (2011). Inteligencia emocional plena: Hacia un programa de regulación emocional basado en la conciencia plena (Disertación doctoral). Universidad de Málaga. Recuperado de http://riuma.uma.es/xmlui/bitstream/handle/10630/5053/Tesis\%20Doctoral\%20 de\%20H\%C3\%A9ctor\%20Arturo\%20Enr\%C3\%ADquez\%20Anchondo.pdf? sequence=1

Etxebarria, I. (2009). Las emociones autoconscientes positivas: El orgullo. En E. G. FernándezAbascal (Coord.). Emociones positivas (pp. 167-180). Madrid: Pirámide.

Fernández-Abascal, E.G. (Coord.). (2011). Emociones positivas. Madrid: Pirámides.

Fernández-Ballesteros, R. (2004). Evaluación psicológica: Conceptos, métodos y estudio de casos. Madrid: Pirámide.

Fernández-Berrocal, P., Berrios-Martos, P., Extremera, N., Augusto, J. M. (2012). Inteligencia emocional: 22 años de avances empíricos. Behavioral Psychology, 20(1), 5-13.

Fernández-Berrocal, P. y Extremera, N. (2002). La inteligencia emocional como una habilidad esencial en la escuela. Revista Iberoamericana de Educación, 29(1), 1-6. Recuperado de http://emotional.intelligence.uma.es/documentos/PDF8habilidad esencial en la escuela.pdf

Fredrickson, B. L. (2013). Positive emotions broaden and build. Advances in experimental social psychology, 47, 1-53. Doi: https://doi.org/10.1016/B978-0-12-407236-7.00001-2

Fregoso, O. A., López, J., Navarro, G. y Valadez, M. (2013). Habilidades emocionales en estudiantes de educación secundaria. Uaricha, 10(23), 89-102. Recuperado de http:// www.revistauaricha.umich.mx/Articulos/uaricha 1023 089-102.pdf

García, A. (2013). Los trastornos emocionales en la infancia y la adolescencia. Su identificación. Alternativascubanasen Psicología, 1(3),80-87. Recuperado de http://acupsi.org/articulo/44/ los-trastornos-emocionales-en-la-infancia-y-la-adolescencia-identificacin.html

Greco, C. (2010). Las emociones positivas: Su importancia en el marco de la promoción de salud en la infancia. Liberabit, 16(1), 81-94. Recuperado de http://www.scielo.org.pe/pdf/liber/ v16n1/a09v16n1.pdf

Guedes, S. M. \& Álvaro, J. L. (2010). Naturaleza y cultura en el estudio de las emociones. RES, 13, 31-47. Recuperado de http://www.fes-sociologia.com/files/res/13/03.pdf

Henao, G. C. y García, M. C. (2009). Interacción familiar y desarrollo emocional en niños y niñas. Revista Latinoamericana de Ciencias Sociales, Niñez y Juventud, 7(2), 785-802. Recuperado de http:// revistaumanizales.cinde.org.co/index.php/Revista-Latinoamericana/article/view/193/89 
doi: http://dx.doi.org/10.15359/ree.21-1.19

URL: http://www.una.ac.cr/educare

CORREO: educare@una.cr

Hernández, R., Fernández, C. y Baptista, M. (2010). Metodología de la investigación (5a ed.). Madrid: McGrau-Hill. Recuperado de https://www.academia.edu/6399195/Metodologia de la investigacion 5ta Edicion Sampieri

Jiménez, M. I. y López, E. (2013). Impacto de la inteligencia emocional percibida, actitudes sociales y expectativas del profesor en el rendimiento académico. Electronic Journal of Research in Educational Psychology, 11(29), 75-98. Recuperado de http://www.redalyc.org/ articulo.oa?id=293125761004

Miguel-Toval, J. J. y Cano-Vindel, A. (2002). Emoción y clínica: Psicopatología de las emociones. En F. Palmero, E. G. Fernández-Abascal, F. Martínez y M. Chóliz (Coords.), Psicología de la motivación y la emoción (pp. 571-581). Madrid: McGraw-Hill.

Oblitas, L. A. (2010). Emociones, salud y enfermedad. Revista Athenea, 1(1), 1-40. Recuperado de https://rinconemocional.files.wor dpress.com/2012/05/emociones-salud-y-enfermedad.pdf

Oros, L. B. (2011). Nuevas contribuciones para el desarrollo de emociones positivas en niños. En M. C. Richaud, V. Lemos (Eds.), Psicología y otras ciencias del comportamiento. Compendio de investigaciones actuales (pp. 63-78). Entre Ríos: Universidad Adventista del Plata.

Pérez, Y. (2014). Particularidades de la regulación de emociones displacenteras en adolescentes con diagnóstico de hipertensión arterial esencial (Tesis de doctorado). Universidad Central Marta Abreu de las Villas. Santa Clara. Recuperado de http://tesis.repo.sld.cu/924/1/Tesis.pdf

Piqueras, J. A., Ramos, V., Martínez, A. E. y Oblitas, L. A. (2009). Emociones negativas y su impacto en la salud mental y física. Suma Psicológica, 16(2), 85-112. Recuperado de http://www. redalyc.org/articulo.oa?id=134213131007

Richaud, M. C. (2010). Gender and cultural patterns of mothers' and fathers' attachment and links with children's self-competence, depression and loneliness in middle and late childhood. En L. A. Newland, H. S. Freeman y D. D. Coyl (Eds.), Emerging topics on father attachment: Considerations in theory, context and development (pp. 53-71). London: Routledge.

Roca, M. A. (2014). ¿Psicología positiva... o una mirada positiva de la psicología? Alternativas cubanas en Psicología, 2(6), 7-15.

Sánchez-Aragón, R. y Díaz-Loving, R. (2009). Reglas y preceptos culturales de la expresión emocional en México: Su medición. University Psychology, 8(3), 793-805. Recuperado de http://revistas.javeriana.edu.co/index.php/revPsycho/article/view/625/386

Vázquez, C. y Hervás, G. (Coords.). (2009). La ciencia del bienestar. Fundamentos de una psicología positiva. Madrid: Alianza. 